\title{
New Developments and Applications of Electron Beam Absorbed Current in SEM
}

\author{
Grigore Moldovan ${ }^{1}$ \\ 1. point electronic $\mathrm{GmbH}$, Halle, Germany \\ * Corresponding author: grigore.moldovan@pointelectronic.de
}

Electron Beam Absorbed Current (EBAC) is a long-discovered operation mode of Scanning Electron Microscopes (SEMs), where the current from the specimen is used as a signal for imaging [1,2]. It has been explored in the early days of electron microscopy, termed as charge collection or specimen current, but it has been largely abandoned within general microscopy in favour of Secondary Electron (SE) detectors because of requirements for higher speed. However, EBAC has retained a core value for Electrical Failure Analysis (EFA), primarily because of its derivative technique of Resistive Contrast Imaging (RCI) [3]. As RCI electronics are developing for improved speed and signal-to-noise ratio, it is now timely to revisit application of EBAC to general SEM work.

Main historical limitation of EBAC has been the requirement to amplify a signal in the range of pA, which was slow and noisy. As one would expect, electronics for low-current high-speed applications are now much more able than they were in the 1960s. It is now routine to work with EBAC imaging conditions similar to those of conventional solid-state BSE detectors. For example, a pixel dwell time in the range of $10 \mu \mathrm{s} /$ pixel may be now achieved for an EBAC gain of $10^{12} \mathrm{~V} / \mathrm{A}$, which matches typical beam currents. This is largely because of the introduction of in situ preamplification and 12-bit digitization. Another key advancement in EBAC has been introduction of fully calibrated amplification and image acquisition, which allows for full signal quantification - this is not possible with SE.

A range of standard samples and selected applications will be used to explore the nature of EBAC contrast. Tin balls (Figure 1) and Au particles (Figure 2) on Carbon are shown here to illustrate that the EBAC contrast more closely resembles that of In-Lens detector, rather than the conventional SE detectors. It is evident that EBAC retains the high-resolution information, similar to In-Lens, as well as the orientation contrast. As described previously [2], EBAC retains the same signal under low magnification and/or long working distance conditions, whilst SE is limited by collection geometry.

Given the combination of independence from SEM geometry, and the addition of signal calibration, EBAC is proposed here as a technique for reliable measurements of local properties. New applications include measurement of surface tilt/normal on samples of uniform composition, measurement of density on samples with flat surfaces, as well as quantification of orientation contrast independent of working distance (Figure 3). A range of further example data and applications will be presented [4].

References:

[1] HJ Leamy, J. Appl. Phys. 53 (6) (1982), p. R51.

[2] J Goldstein et al., in "Scanning Electron Microscopy and X-Ray Microanalysis", (Singer, US). DOI 10.1007/978-1-4615-0215-9

[3] CA Smith et al., IEE Transactions on Electron Devices, ED-33 (2) (1986), p. 282.

[4] The author acknowledges design of EBAC electronics by Wolfgang Joachimi and Uwe Grauel. 
(a)

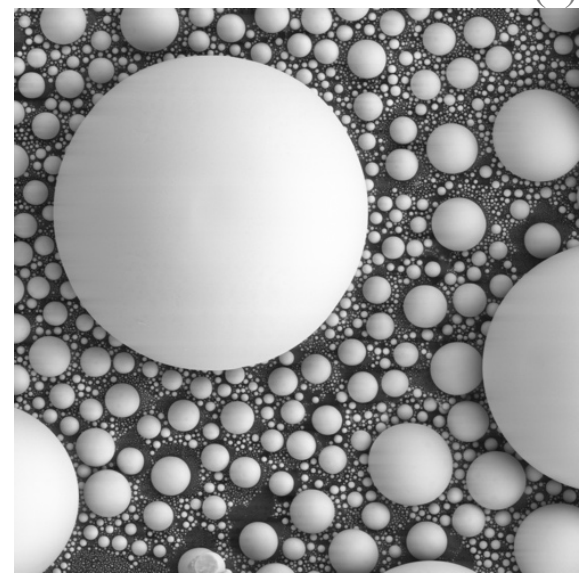

(b)

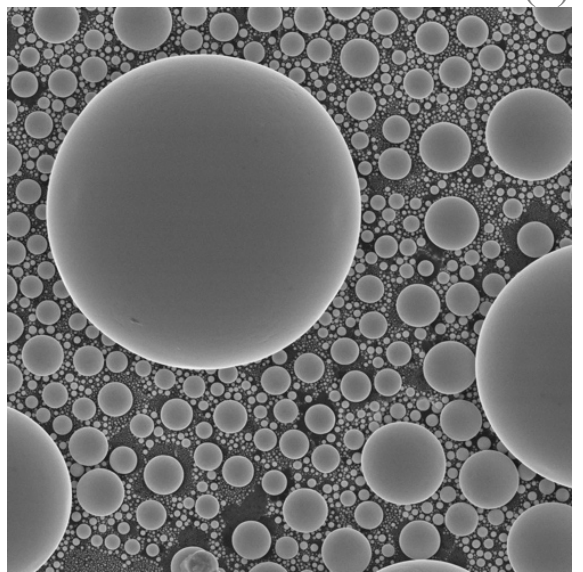

(c)

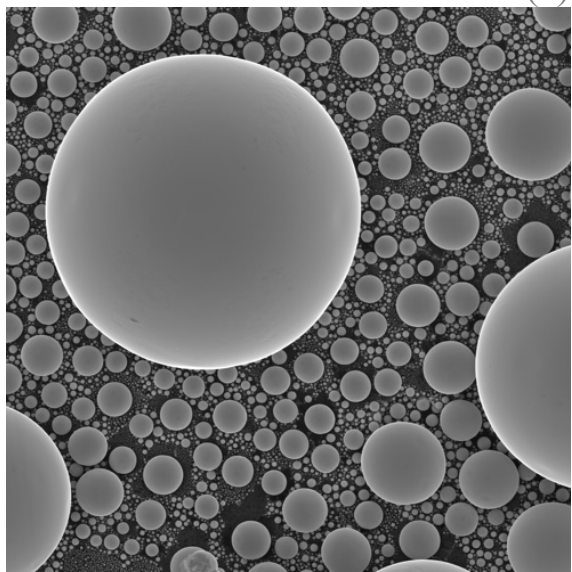

Figure 1. Comparison of conventional SE (a), In-Lens (b) SE and EBAC(c) micrographs of Tin balls on Carbon. Imaging conditions are $10 \mathrm{kV}, 10 \mathrm{~mm}$ working distance, $43.47 \mu \mathrm{m}$ field of view.
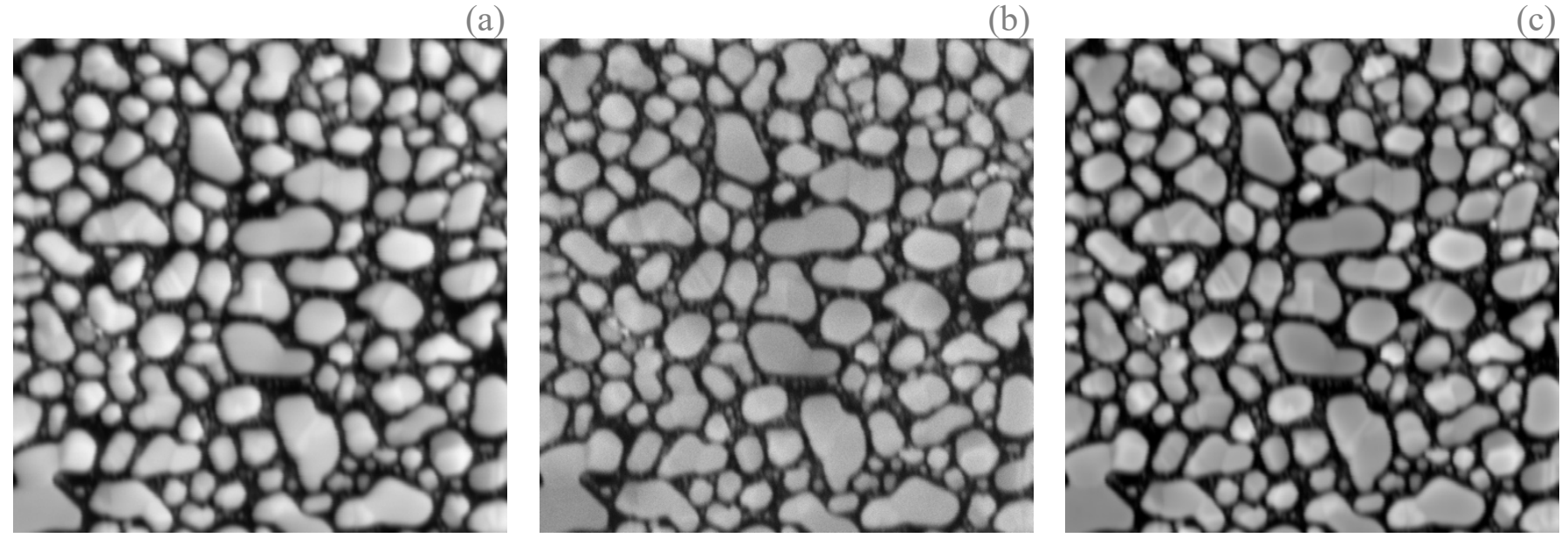

Figure 2. Comparison of conventional SE (a), In-Lens (b) and EBAC (c) micrographs of Au particles on Carbon. Imaging conditions are $10 \mathrm{kV}, 10 \mathrm{~mm}$ working distance, $2.608 \mu \mathrm{m}$ field of view.
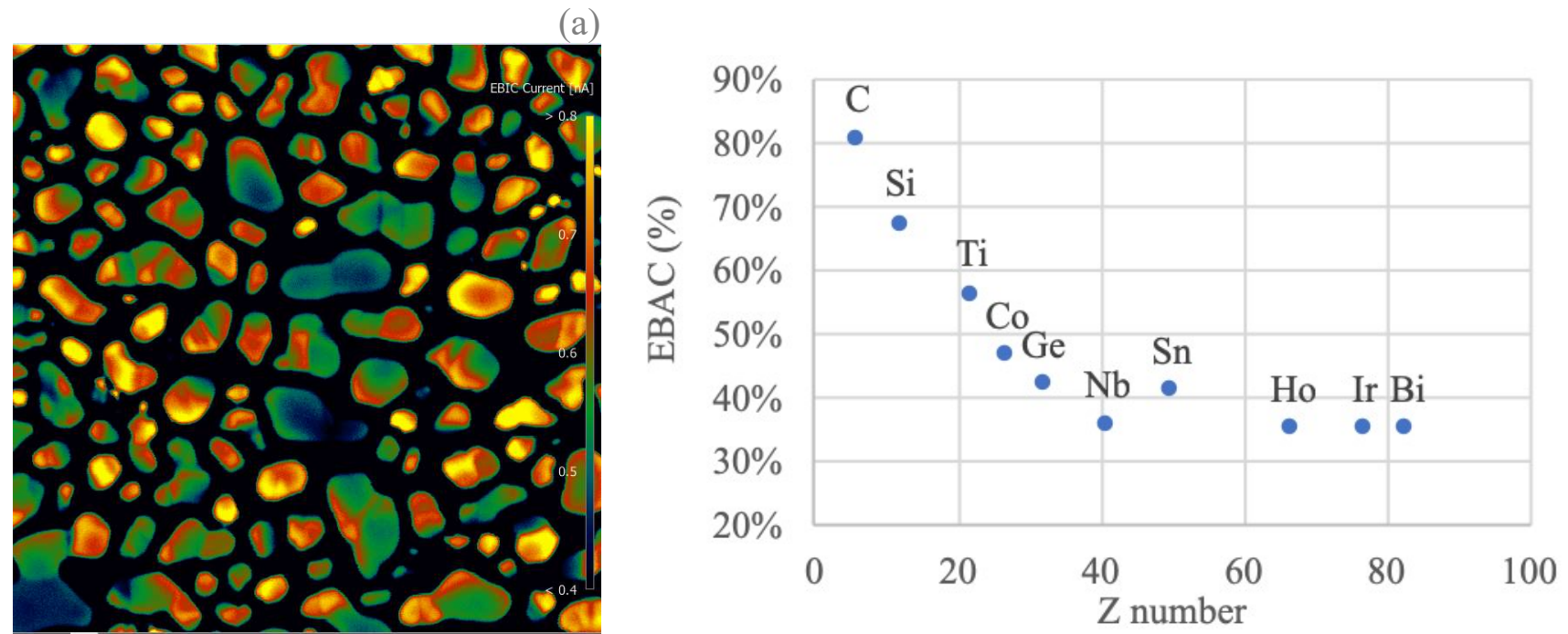

Figure 3. Highlights of quantified data, showing EBAC values of Au particles on Carbon (a) and measured absorbed/beam current ratios (c). Measurement of Sn has a high error due to uneven surface. 